\title{
Transitionen im Lebenslauf - mit Zoom auf die Familie \\ R. Levy
}

\section{Transition zur Elternschaft - Retraditionalisierung der Familie}

Nach der Exposition eines theoretischen Zugangs zur soziologischen Analyse von Lebensläufen stellt dieser Text eine empirische Analyse von Lebensläufen zwischen Beruf und Familie in der Schweiz vor und endet mit einer kritischen Sichtung des Phänomens der Retraditionalisierung der Familienstruktur beim Übergang zur Elternschaft sowie der institutionellen Förderungsbedingungen dieser "Rückwärtsentwicklung".

Aufgrund eines struktursoziologischen Ansatzes zur Lebenslaufanalyse wird erläutert, weshalb Statusübergänge als potentielle Bruchstellen im Lebensverlauf besonders wichtig sind. Auf diesem Hintergrund wird als Illustration der folgenreiche Übergang von Paaren zur Elternschaft mittels Optimal Matching analysiert ${ }^{1}$ und anhand einer qualitativen Folgestudie näher beleuchtet. Die Resultate werfen die Fragen danach auf, inwiefern die gefundenen Verlaufsformen sozial konditioniert sind und welche Rolle das Geschlecht und seine Handhabung durch soziale Institutionen dabei spielt.

\section{Ein soziologischer Ansatz zur Analyse von Lebensläufen}

\subsection{Wie kann man Lebensläufe soziologisch thematisieren?}

Wo kann man ansetzen, wenn man sich zum Ziel setzt, den soziologisch relevanten Anteil am Zustandekommen von Lebensläufen so klar als möglich zu konzeptualisieren? Mindestens drei Ausgangspunkte werden durch die bestehende Literatur nahegelegt:

1. Soziale Vorstellungen und Deutungsmuster, z.B. über die Zuschreibung der Verantwortung für seine Lebensführung. Nach Kohli (1985) ging eine der drei grossen Tendenzen der vergangenen 200-300 Jahre in Richtung zunehmender Biographisierung: in der Kultur der west-

\footnotetext{
'Die Methode des Optimal matching erlaubt den systematischen Vergleich von Sequenzen - irgendwelcher Art, nicht nur im Sinn chronologischer Abfolgen. Sie stammt aus der Genomik und wird seit einigen Jahren zunehmend auch in den Sozialwissenschaften verwendet, wenn es um die Analyse von Longitudinaldaten geht (Aisenbrey 2000). Optimal matching vergleicht sämtliche zu analysierenden verwendet, wenn es um die Analyse von Longitudinaldaten geht (Aisenbrey 2000). Optimal matching vergleicht sämtliche zu analysierenden
Sequenzen paarweise und drückt ihre Unterschiedlichkeit als Distanz aus. Diese Distanzen können ihrerseits cluster-analysiert werden, was die Identifikation allfälliger Sequenz- oder Verlaufstypen erlaubt. So ermittelte Typen können in einem weiteren Schrit regressionsanalytisch untersucht werden, was ermöglicht, nach Faktoren zu suchen, welche das unterschiedliche Auftreten der gefundenen Typen erklären können.
} 
lichen Gesellschaften hat im Verlauf der Modernisierung die Vorstellung überhand genommen, jeder Mensch sei für den Verlauf seiner Biographie grundsätzlich selbst verantwortlich. Dieser Idee entspricht auch das in den letzten rund 20 Jahren gestiegene soziologische Interesse an der individuellen "Agency", d.h. an der Gestaltungsmacht individueller Akteure gegenüber sozialen Determinismen oder Zwängen kultureller oder struktureller Art. Wer solchen Vorstellungen anhängt, so ginge die Argumentation unter diesem Gesichtspunkt, versucht auch, sie biographisch zu realisieren, sodass sich diese gesellschaftlichen Vorstellungen über individuelle Umsetzungspraxis in effektive Verläufe übersetzen.

2. In der Kultur vorherrschende normative Überzeugungen über den normalen oder "richtigen" Verlauf des Lebens, namentlich in Form von Altersnormen, wie sie seit Neugarten et al. (1965) besonders in der Sozialpsychologie häufig untersucht werden, aber auch Normen darüber, welche Tätigkeiten im Leben wichtig sind und in welcher Abfolge sie normalerweise stehen sollten. Auch hier ginge die Argumentation in die Richtung, dass gesellschaftlich vorherrschende Normen durch individuelle Akteure in Verhalten umgesetzt werden, weil sie dazu tendieren, zu diesen Normen konform zu sein.

3. Neben diesen kulturellen Aspekten kommen aber auch die effektiven Verläufe als Ansatzpunkt in Frage, also das faktische Verhalten. Auch in diesem Fall könnte die Frage nach allfälligen Übereinstimmungen der Verläufe durch den Bezug auf zugrunde liegende gemeinsame Normen oder allgemeiner geteilte kulturelle Vorstellungen beantwortet werden, als Grundlage einer zweiten Erklärungsstrategie bietet sich jedoch auch das strukturelle Umfeld der Individuen an, welches ihre Verhaltensbahnen durch die - weitgehend institutionelle Strukturierung ihrer Lebensumwelt kanalisiert.

"Kulturalistische" Interpretationen des Typs 1 und 2 erscheinen oft als naheliegend und sind auch in der Soziologie entsprechend beliebt. Wie die empirische Forschung zeigt, greifen sie jedoch häufig zu kurz bzw. neben das Ziel, weil Individuen wegen ihrer Einbettung in soziale Zusammenhänge nicht immer aufgrund ihrer normativen Überzeugungen handeln (können), sondern eher aufgrund der Möglichkeiten und Unmöglichkeiten (opportunity structures), die sie in ihrem Umfeld vorfinden, was sozialstrukturelle Erklärungsansätze in den Vordergrund rückt. Dies bedeutet nicht, dass kulturelle Phänomene soziologisch uninteressant wären, weist aber darauf hin, dass Normen und Werte häufig Verhalten nicht in einem psychologischen bzw. kausalen Sinne motivieren und insofern erklären, sondern eher im Nachhinein zur Begründung von Verhalten herangezogen werden. Im Folgenden soll deshalb das Schwerge- 
wicht auf den dritten Zugang gelegt werden. Wie kann der allfällige Zusammenhang zwischen individuellen Verläufen und Gesellschaftsstruktur konzeptuell gefasst werden? ${ }^{2}$

\subsection{Ein struktursoziologischer Ansatz: Lebenslauf als Bewegung durch den sozialen Raum}

Als zentrales Element zur Bestimmung und Analyse der Beziehung individueller Akteure zur Gesellschaft erscheint in struktursoziologischer Sicht deren Verortung im sozialen Raum; der Lebensverlauf kann folgerichtig als Bewegung durch diesen Raum gefasst werden. Damit stellt sich die Frage, wie dieser Raum und individuelle Orte darin zu konzeptualisieren sind. Um diese Frage jedenfalls in einem ersten Schritt möglichst einfach zu beantworten, kann von einer Sichtweise ausgegangen werden, die den sozialen Raum einer Gesellschaft als eine Gesamtheit von gegeneinander abgegrenzten Feldern sozialer Interaktion behandelt. ${ }^{3}$ Mit Blick auf moderne Gesamtgesellschaften ist namentlich an institutionelle Sektoren zu denken wie Bildung, Berufswelt, Politik, Familie usw. All dies sind organisierte Handlungs- und Interaktionsfelder, an denen Individuen regelmässig teilnehmen.

Dabei fällt schnell auf, dass im zeitlichen Ablauf die Teilnahme an solchen Feldern sequentiell oder auch synchron erfolgen kann; beides ist lebenslaufanalytisch wichtig, wie gleich noch weiter auszuführen sein wird.

Wie steht es mit der Verortung von Akteuren in solchen Feldern? Dafür kann auf klassische und elementare soziologische Konzepte wie Teilnahme, Status, Position oder Rolle zurückgegriffen werden (Linton 1936, Merton 1957). Drei Aspekte von Status erscheinen dabei als wichtig: Teilnahme, Stellung und Rolle. Status wird hier in einem allgemeinen Sinn verwendet, Position oder Stellung sowie Rolle in einem spezifischeren. An einem sozialen Feld teilzunehmen heisst in der Regel, eine bestimmte Stellung oder Position in dessen innerer Struktur zu besetzen und mit mehr oder weniger Konformität die mit der Position verbundene Rolle (im Sinn einer zusammenhängenden Gesamtheit von Erwartungen) zu übernehmen. Diese Trias von Aspekten gilt im Prinzip für jedes soziale Feld, an dem teilgenommen wird, wenn sie sich auch je nach den Besonderheiten des jeweiligen Feldes anders darstellen können.

\footnotetext{
${ }^{2}$ Dies sind nicht die einzig denkbaren theoretischen Ansätze. Weitere könnten etwa darin bestehen, Lebensläufe als das Zusammentreffen ontogenetischer Entwicklungsgesetzen aufzufassen, etwa im Sinn von Piagets Entwicklungsstufen, oder gar als Ergebnis des Aufeinandertreffens genetischer Programmierung mit sozialen Zufällen (zB. wo jemand an welchem Zeitpunkt steht). Thnen soll hier nich weiter nachgegangen werden.

${ }_{3}^{3}$ Die Rede von Feldern sozialer Interaktion kann auf den österreichischen Sozialpsychologie-Pionier Kurt Lewin zurückgeführt werden, de Die Rede Elen Begiff a grerer

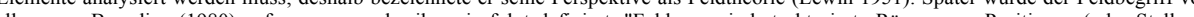
allem von Bourdeu (1980) aufgenommen, der hn wie fogt definit. " Felder ... sind strukturiete Raume von Positionen (oder Stellen), deren Eigenschaften von ihrem Ort im Raum abhängen und die unabhängig von den Eigenschaften der sie besetzenden Akteure analysier werden können, welch letztere im übrigen teilweise durch sie bestimmt werden." (1980, Uebersetzung RL). Auch der Schweizer Soziologe Peter Heintz verwendet (1968) einen wenn auch weniger anspruchsvoll und voraussetzungsreich definierten Feldbegriff, von dem hier ausgegangen wird.
} 
Berücksichtigt man weiter, dass zumindest Erwachsene meistens an mehreren sozialen Feldern zugleich teilnehmen, so ergibt sich, dass die strukturelle Verortung einer Person durch ihr Statusprofil bzw. ihre Statuskonfiguration gegeben ist, also durch so viele Teilnahme + Stellung + Rolle-Kombinationen wie die Zahl der Felder, an denen sie teilnimmt. Durch das Statusprofil ist die Stellung der Person im gesellschaftlichem Schichtungssystem gegeben, aber auch der Umfang und der Grad an Vollständigkeit ihrer sozialen Teilnahme. Beispiele unvollständiger Statusprofile für Erwachsene in Gegenwartsgesellschaften sind etwa Arbeitslosigkeit, d.h. man nimmt an der Arbeitswelt entgegen den vorherrschenden sozialen Normen und Zwängen nicht teil; als diesbezüglich nicht statusunvollständig, obwohl nicht in die Arbeitswelt integriert, gelten dagegen Kinder, Pensionierte, schwer Kranke oder Häftlinge. Mit diesem Konzept kann der Lebenslauf, also die biographische Bewegung durch den gesellschaftlichen Raum, folgerichtig als Sequenz oder Abfolge der von einem Individuum innegehabten Statusprofile gefasst werden.

\subsection{Institutionalisierung von Lebensläufen}

Zur sozialen Organisation oder Institutionalisierung von Lebensläufen wäre vieles zu sagen (siehe namentlich Krüger 2001), hier nur so viel: Institutionen im Sinn real funktionierender Organisationen - und nicht nur normativer Grundvorstellungen - spielen eine zentrale Rolle in der Strukturierung von Lebensläufen. Beispiele: der obligatorische Schulbesuch konstituiert einen wichtigen Teil von Kindheit und Adoleszenz; die Ausrichtung der Ausbildung auf die Berufswelt (besonders deutlich im Fall der Berufsbildung) bereitet den Eintritt in diese vor; das öffentlich geregelte Alterssicherungssystem reguliert den Austritt aus der Berufswelt, und zwar nach Modalitäten, die z.T. davon abhängen, wie die Erwerbsteilnahme gestaltet war; die Familie als einer der wichtigsten Orte der Lebenslaufverflechtung ist das Koordinationszentrum der unterschiedlichen und oft divergierenden zeitlichen, ressourcenmässigen und planerischen Imperative der diversen sozialen Felder, an denen ihre Mitglieder - Mutter, Vater, Kinder, allenfalls pflegebedürftige weitere Personen - teilnehmen. All dies hat vielfältige und oft weitreichende Einflüsse auf die Lebensläufe der beteiligten Individuen; in dem Masse, wie Geschlecht als hochgeordneter Masterstatus in praktisch all diese institutionellen Teillogiken von vornherein eingelassen ist, sind sie auch alle an einem strukturellen Doing gender beteiligt, das weit über das Niveau der zwischenmenschlichen Interaktionen hinausgeht. ${ }^{4}$

${ }^{4}$ Das zur Analyse von Geschlechtstypisierungen zentrale Konzept des Doing gender (West und Zimmerman 1987) wird meistens auf interaktive Performanz bezogen, also auf zwischenmenschliche Interaktionen, in denen Geschlechtervorstellungen dargestellt, reproduzier und verfestigt werden. Demgegenüber wird hier unterstrichen, dass auch organisierte Institutionen in ihrem gewöhnliches Funktionieren meist unausgesprochenen oder sogar "ungedachten" Normalitätsvorstellungen über Genderrollen bzw. gendertypisch organisierte Verhaltensund Organisationsweisen - etwa von Familien - voraussetzen und diese dadurch ihren BenutzerInnen auch aufdrängen. Dies wird hier als 


\section{Statusübergänge: Bruchstellen im Lebenslauf}

Im Rahmen der eben vorgestellten Konzeptualisierung stellen Statusübergänge Veränderungen des Statusprofils dar. Darunter kann aber Verschiedenes subsumiert werden, denn grundsätzlich, gewissermassen rein logisch gesehen, können sich im biographischen Verlauf alle Aspekte eines Statusprofils verändern, einzeln oder kombiniert: die Teilnahme an einem sozialen Feld kann aufgenommen oder aufgegeben werden, die Stellung darin kann durch vertikale Mobilität verbessert oder verschlechtert werden, in Abhängigkeit davon, aber auch aus anderen Gründen können sich auch die zugehörigen Rollen in ihren vielfältigen Aspekten verändern. Ein wichtiger Unterschied zwischen diesen drei Möglichkeiten besteht darin, dass die Veränderung der Teilnahme deshalb als besonders folgenreich erscheint, weil sie automatisch auch Veränderungen von Position und Rollen nach sich zieht. Diese Besonderheit wird dann noch weiter verstärkt, wenn sich mehrere Teilnahmen gleichzeitig oder in kurzer Abfolge verändern, wie dies beispielsweise beim Übergang von der Jugendlichenphase in die junge Erwachsenenphase der Fall ist (namentlich Übertritt von der Ausbildung ins Berufsleben, Aufnahme eines eigenen Domizils, Aufnahme oder Konsolidierung einer Partnerschaft, Übergang zur Elternschaft - vgl. dazu schon Blancpain und Häuselmann 1974).

Neben Veränderungen seiner einzelnen Komponenten kann sich das Statusprofil auch gesamthaft in der sozialen Schichtung aufwärts oder abwärts bewegen, und es kann durch zusätzliche Teilnahmen ausgeweitet oder durch Wegfall von Teilnahmen eingeschränkt werden. Diverse Studien weisen darauf hin, dass die Anzahl der Teilnahmen eigenständige Auswirkungen auf das Wohlbefinden erwachsener Menschen hat (von der subjektiven Zufriedenheit bis hin zur Gesundheit und zur Lebenserwartung), wobei es anscheinend ein Optimum gibt (Thoits 1986).

Strukturelle Veränderungen in sozialen Feldern können individuelle, aber auch kollektive Veränderungen von Statusprofilen nach sich ziehen und damit direkt Lebensläufe beeinflussen (Beispiel: wirtschaftliche Rezession mit Arbeitslosigkeit,). Theoretisch wichtig ist hier die direkte Anschlussmöglichkeit zwischen gesellschaftlichem Strukturwandel und individuellen Lebensläufen, die durch die Konzeptualisierung des Lebenslaufs als Sequenz von Statusprofilen hergestellt wird.

Ausgehend von den vorhergehenden Überlegungen lässt sich eine allgemeine Hypothese formulieren: Statusübergänge sind für die Identität (für die selbst empfundene ebenso wie auch 
die von anderen wahrgenommene), für soziales Lernen und für die Lebensqualität (als Quellen sozialer Anerkennung und subjektiver Zufriedenheit) wichtiger als die Phasen dazwischen, weil die personale und soziale Identität stark vom Profil der sozialen Teilnahme abhängt - und dieses Profil verändert sich typischerweise bei Statusübergängen. Phasen in Lebensläufen dürften deshalb eher die Konsolidierung von Identitäten und informellen Beziehungen fördern, Statusübergänge (stärker als beispielsweise "blosse" Positionsveränderungen) dürften Identitätsveränderungen provozieren und Lernprozesse dynamisieren. ${ }^{5}$

Nicht alle Partizipationen sind gleich gewichtig. Ihr Gewicht dürfte von personalen und sozialen Werten abhängen (u.a. von direkt lebenslaufbezogenen Normen: wann und in welcher Reihenfolge haben Übergänge zu erfolgen, etwa jener zum Erwachsenenalter), aber auch und vermutlich noch stärker von ihrer institutionellen Gewichtung und strukturierenden Kraft. Als besonders zentral können die Berufstätigkeit und die Familie gelten, vermutlich sind sie deshalb auch besonders folgenreich. Die Zentralität der Berufstätigkeit ergibt sich zwar auch aus deren sozialer Wahrnehmung als zentral, aber in den Gegenwartsgesellschaften noch stärker aus dem strukturellem Link zwischen Berufstätigkeit und Geld verdienen, weil Geld in einer Marktwirtschaft lebensnotwendig ist und nicht ohne weiteres anders als durch Erwerbstätigkeit beschafft werden kann. Die Zentralität der Familie resultiert vermutlich stärker aus deren affektiv stabilisierender Funktion, jedenfalls für ihre erwachsenen Mitglieder. Wir werden in der Folge sehen, dass darüberhinaus das Gewicht eines und desselben Feldes nicht unbedingt für alle Individuen gleich ist.

Eine weitere Nuancierung ist noch zu erwähnen: inwiefern ist etwa ein Schritt sozialer Aufwärtsmobilität als Statusübergang zu werten? Dies hängt von verschiedenem ab: von der durch den Aufstieg überwundenen vertikalen Distanz (ist es ein kleiner oder ein grosser hierarchischer Schritt - grössere Schritte haben weiter gehende Konsequenzen, etwa im Sinne des Wechsels in eine anderes soziokulturelles Milieu, einer markanten Kaufkraftveränderung o.ä., und damit eher den Charakter eines Statusübergangs), vom damit allenfalls verbundenen Wechsel der Organisation (andere Firma, anderer Arbeitgeber) oder des Ortes (mit Folgen für das Aufrechterhalten des privaten Beziehungsnetzes), aber auch vom Gewicht des betroffenen Feldes (dieses Gewicht dürfte im Fall eines Aufstiegs in der Pfadfinderhierarchie, im informellen Freundeskreis oder im Betrieb bei weitem nicht dasselbe sein). Mit anderen Worten hängt die Beantwortung der Frage von verschiedenem ab - man darf also nicht davon ausge-

${ }^{5}$ Wobei selbstverständlich nicht alle Statusübergänge gleich folgenreich sind. Auch hierzu kann eine Reihe von Faktoren identifiziert werden, die vermutlich das faktische Gewicht von Statusübergängen für die betroffenen Personen beeinflussen, dies kann aber aus Raumgründen nicht ausgeführt werden. Das Gewicht von Partizipationen ist nur einer dieser potentiellen Faktoren. 
hen, es handle sich dabei um einfache, dichotome Erscheinungen. Die vorgestellten Konzepte können deshalb nicht mechanisch angewendet werden.

\section{Zoom auf den Übergang zur Elternschaft und die Lebenslauf- standardisierung}

Da bis in die 90er Jahre noch sehr wenig longitudinale Lebenslaufanalysen vorlagen, bestand ein weites Feld für Hypothesen und Behauptungen über die Sachlage, namentlich über den Grad an sozialer Standardisierung der Lebensläufe. ${ }^{6}$ Diesbezüglich wurden während längerer Zeit drei Hauptthesen vertreten und zum Teil auch gegeneinander verteidigt.

Am frühesten wurde die These aufgestellt, dass es in westlichen Gesellschaften zwei geschlechtsspezifische Standard- oder Normalbiographien gibt, eine männliche, lediglich berufsbezogene, und eine weibliche, die zugleich durch Berufs- und Familienteilnahme strukturiert wird (Held und Levy 1974, Levy 1977).

Kohli $(1985,1986)$ hat demgegenüber die Vorstellung vertreten, es gebe ein zentrales, durch den Dreischritt Bildung - Berufstätigkeit - Rentnerleben organisiertes Hauptmodell, von dem einfach verschiedene Abwandlungen vorkämen.

Mit dem Aufkommen der Thesen über die zweite Modernisierung bzw. die postmoderne Gesellschaft (Lyotard 1979, Beck 1986, Giddens 1991, Bauman 2000) wurde als dritte Antithese das Postulat bekannt, neben vielen anderen sozialen Phänomenen (zu denen oft auch die soziale Schichtung gezählt wurde, etwa bei Hradil 1990) verlöre sich in der "Spätmoderne", also in den Gegenwartsgesellschaften, auch die Standardisierung der Lebensläufe (Beck und BeckGernsheim 1994). ${ }^{7}$

Drei und nicht nur zwei einander ausschliessende Thesen sind eine seltene Ausgangslage für die empirische Abklärung des Sachverhalts und machen diese besonders interessant.

\footnotetext{
${ }^{6}$ Obwohl die empirische Soziologie von Lebensläufen auf den Beginn des 20. Jahrhunderts zurückgeht - oft wird die Studie von Thomas \& Znaniecki (1918) als erste genannt - sind Längsschnittstudien wie etwa jene von Elder (1974) lange seltene Ausnahmen geblieben. Im deutschen Sprachraum fallen die ersten grösseren derartigen Erhebungen in die späten 80er Jahre: die Deutsche Lebensverlaufsstudie des Max-Planck-Instituts (1980-2004, Diewald et al. 2006), das Sozioökonomische Panel (SOEP, http://www.diw.de/de) und diverse Projekte des Sonderforschungsbereichs 186 der DFG in Bremen über "Statuspassagen und Risikolagen im Lebensverlauf. Institutionelle Steuerung des Sondivile Hene (1988-2001, Sackmn \& Wing und individuelle Hante Klolle 2001). E) dernath et al. 1989). Seither entstanden einige grossere Panelunersuchungen we TREe (htp://ree.unibas.ch//home/) oder das Schweizerische Haushaltpanel (http://www.swisspanel.ch/?lang=de). Auch die SAKE des Bundesamts für Statistik wird als Panel geführt, wenn auch al rotierendes, bei dem die Verbleibdauer der Individuen maximal 5 Jahre beträgt.

7 Dass hier fast ausschliesslich deutschsprachige Publikationen zitiert werden, ist kein Zufall. Diese Debatte wurde vor allem und besonders intensiv in der deutschsprachigen Soziologie geführt. Im übrigen hat Kohli später (2003) die Richtigkeit der These geschlechtsspezifischer Verläufe anerkannt.
} 


\subsection{Empirische Lebenslaufanalyse}

Eine der ersten umfassenden empirischen Studien zur Überprüfung dieser Thesen wurde in der Schweiz angestellt. Widmer et al. (2003a) analysierten retrospektive Daten, die bei einem Teil der für die Studie "Couples contemporains" (Widmer et al. 2003b) im Winter 1998-99 befragten Paare erhoben wurden (N=677 für Männer, 670 für Frauen). Levy et al. (2006) replizierten diese Analyse mit Daten des Schweizerischen Haushaltspanels (SHP), das nicht nur Paare umfasst, sondern die gesamte Bevölkerung ( $\mathrm{N}=1696$ für Männer, 1935 für Frauen; nur Personen von mindestens 30 Jahren wurden einbezogen, um eine zur Identifikation von Verlaufstypen genügende Beobachtungsdauer sicherzustellen). Beide Analysen ergaben dieselben Resultate, aber mit unterschiedlichen Frequenzen; die Ergebnisse der zweiten Analyse werden hier kurz vorgestellt. Für Männer und Frauen wurden separat die Lebensläufe in Bezug auf ihre Berufs- und Familienteilnahme für die Altersspanne 16-64 rekonstruiert, wobei den Informationen über Berufstätigkeit insofern die Priorität gegeben wurde, als - aufgrund anderweitiger empirischer Resultate - bei verheirateten oder in Partnerschaft lebenden Personen die Familienarbeit als gegeben, aber als durch die Berufstätigkeit potentiell eingeschränkt betrachtet wurde (eine differenzierte Erfragung etwa der wöchentlich für Familienbelange aufgewendeten Stundenzahl ist retrospektiv undurchführbar). Aufgrund der erhobenen Informationen (retrospektives Modul der Befragungswelle 2002 des SHP) wurden die Lebensläufe als Sequenzen von vorwiegenden Zuständen rekonstruiert, die für jedes Lebensjahr eines Individuums eine von sieben Bedeutungen haben konnten: in Ausbildung, vollzeitig erwerbstätig, teilzeitig erwerbstätig, Familienarbeit, positiver Erwerbsunterbruch (Sabbatical, längere Reise), negativer Erwerbsunterbruch (Arbeitslosigkeit, Krankheit), RentnerIn. Die so rekonstruierten individuellen Verläufe wurden für Männer und Frauen getrennt ${ }^{8}$ mittels Optimal Matching paarweise miteinander verglichen und das Ergebnis (Distanzen in allen Paaren individueller Verläufe) einer Clusteranalyse unterzogen, welche erlaubte, Verlaufstypen empirisch zu ermitteln. Anschliessend wurde mittels Regressionsanalyse exploriert, welche Faktoren zwischen den gefundenen Verlaufstypen differenzieren. Die Darstellung in den sechs folgenden Graphiken gibt für jeden Verlaufstyp eine Synthese; die biographischen Zustände aller dem Typ zugeordneten Individuen werden für jedes Lebensjahr als Prozentverteilung in

${ }^{8}$ Diese Analysestrategie wurde der Alternative, männliche und weibliche Verläufe zusammen zu analysieren, deshalb vorgezogen, weil sie allfällig vorhandene Geschlechtsspezifitäten der Verläufe deutlicher hervortreten lässt. Berücksichtigt sind alle Erwachsenen ab 30 Jahren. 
einem Histogramm aufgetragen. Lesebeispiel aus Abb. 1: die 18-jährigen Männer des Verlaufstyps "Vollzeiterwerb" sind zu 29\% vollzeiterwerbstätig.

Abb. 1 Männliche Verläufe, Typ Vollzeiterwerb

Fig. 1 Vollzeiterwerbsverläufe, Männer $(72 \%, N=1219)$

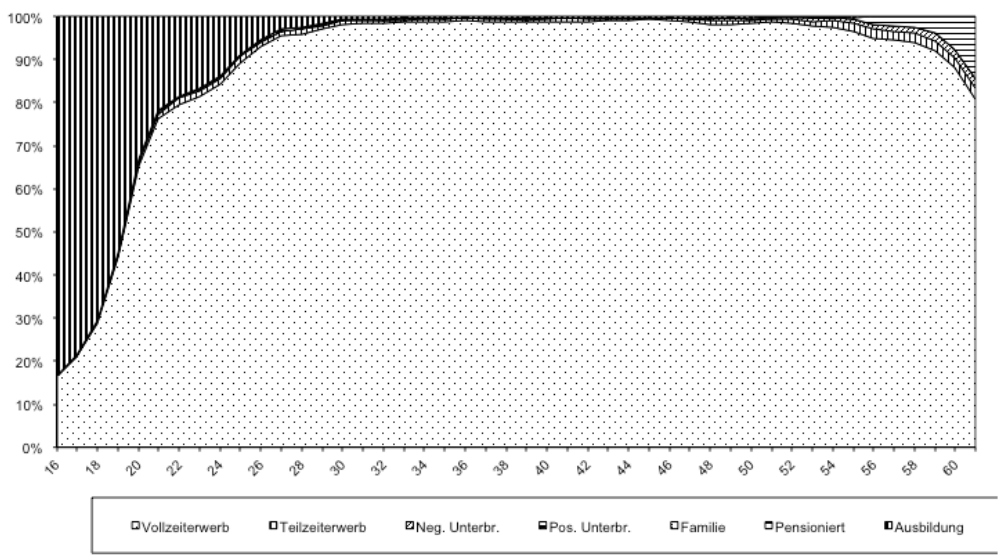

Wie Abb. 1 zeigt, folgen die männlichen Verläufe zu knapp drei Vierteln (72\%) dem Dreischrittmodel, wie es von Kohli $(1985,1986)$ postuliert wurde. Vorherrschende Familienarbeit kommt bei diesem Verlaufstyp so gut wie nicht vor, wir nennen diesen Typ Vollzeiterwerbsverlauf. Die zweite Verlaufsgruppe (Abb. 2) zeigt kein klar profiliertes Muster und ist deshalb nicht als eigentlicher Verlaufstyp, sondern als Restkategorie anzusprechen, die sehr diverse, aber vom Hauptmuster klar abweichende Verläufe umfasst; wir sprechen deshalb von Erratischen Verläufen. 
Abb. 2 Männliche Verläufe, Erratisch

Fig. 2 Erratische Verläufe, Männer $(28 \%, \mathrm{~N}=477)$

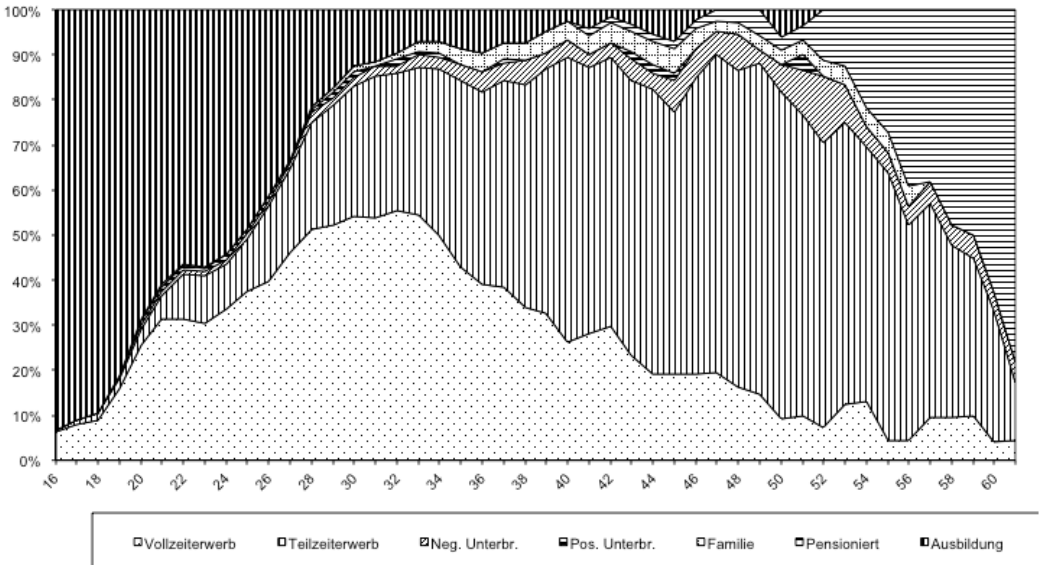

Die Analyse der weiblichen Verläufe zeitigt ein anderes Bild mit vier klar profilierten Verlaufstypen. Diese vierfache Typologie gruppiert sämtliche weiblichen Verläufe, ohne Restkategorie.

Ein erster, der einen Drittel (34\%) der Verläufe umfasst (Abb. 3), entspricht einigermassen dem bei Männern vorherrschenden Modell des durchgehenden Vollzeiterwerbsverlaufs, obwohl Familientätigkeit vorübergehend in deutlich grösserem Ausmass auftaucht als bei Männern. 
Abb. 3 Weibliche Verläufe, Typ Vollzeiterwerb

Fig. 3 Vollzeiterwerbsverläufe, Frauen $(34 \%, \mathrm{~N}=662)$

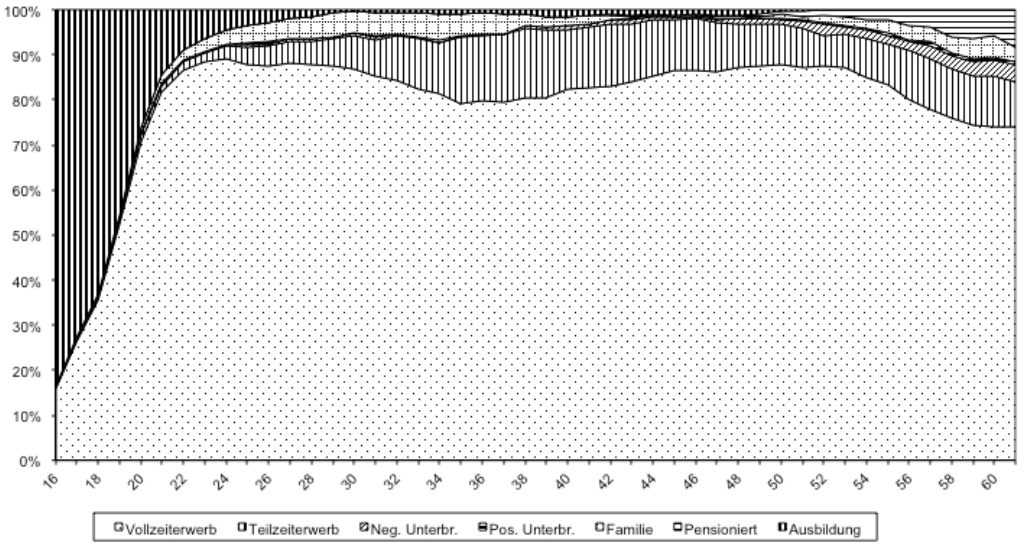

Ein zweiter Verlaufstyp (Abb. 4) entspricht dem traditionell weiblichen Modell, nach dem die Frauen nach anfänglicher Vollzeiterwerbstätigkeit relativ rasch und vor allem definitiv die Berufswelt verlassen und sich gänzlich der Familienarbeit widmen. 13\% der weiblichen Verläufe entsprechen diesem Typ, den wir Familienverlauf nennen.

\section{Abb. 4 Weibliche Verläufe, Typ Familie}

Fig. 4 Familienverläufe, Frauen $(13 \%, N=245)$

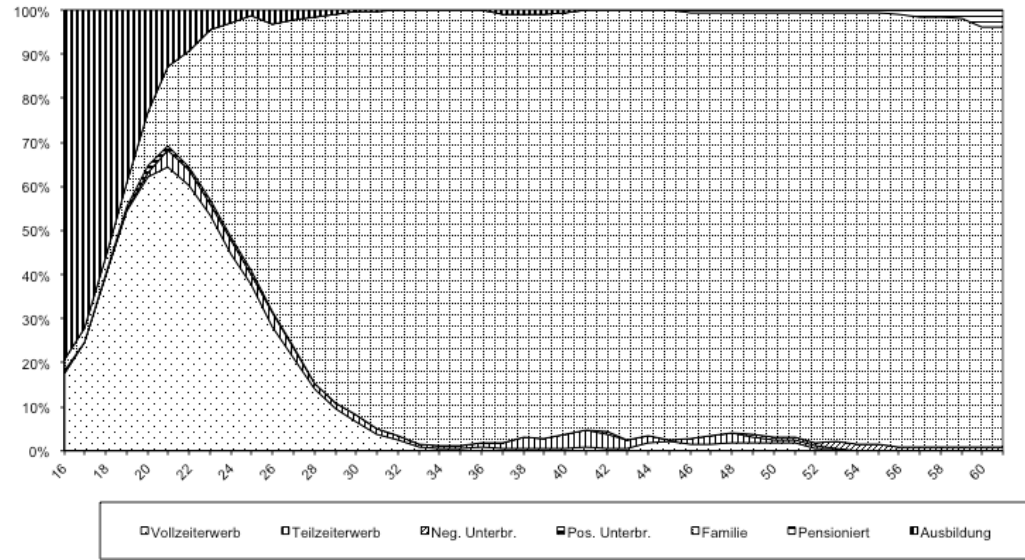

Ein dritter Typ enthält statt dem Rückzug aus der Berufswelt die definitive Reduktion der Erwerbstätigkeit auf Teilzeit (Abb. 5, 23\%), ohne nennenswerte Unterbrechung der Berufsarbeit, wir sprechen von Teilzeiterwerbsverlauf. 


\section{Abb. 5 Weibliche Verläufe, Typ Teilzeit}

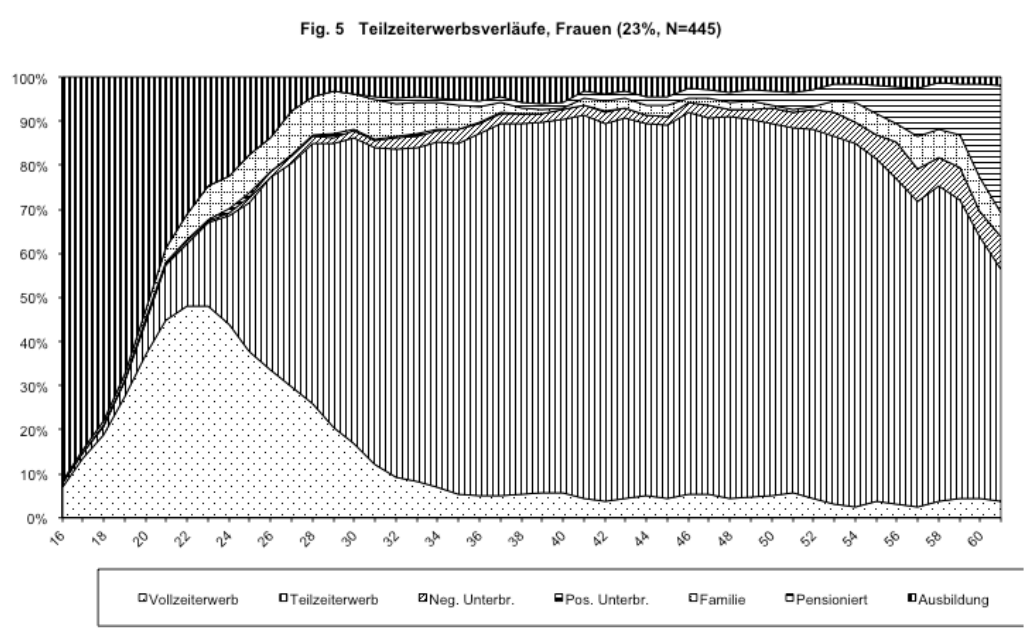

Der vierte Verlaufstyp zeigt ein Hin-und-Her-Muster, nach dem die Frauen zunächst ihre Erwerbstätigkeit aufgeben, sie später aber wieder aufnehmen, allerdings nur teilzeitlich (Abb. 6, 30\%), wir nennen diesen Typ Rückkehr.

\section{Abb. 6 Weibliche Verläufe, Typ Rückkehr}

Fig. 6 Rückkehrverläufe, Frauen $(30 \%, \mathrm{~N}=583)$

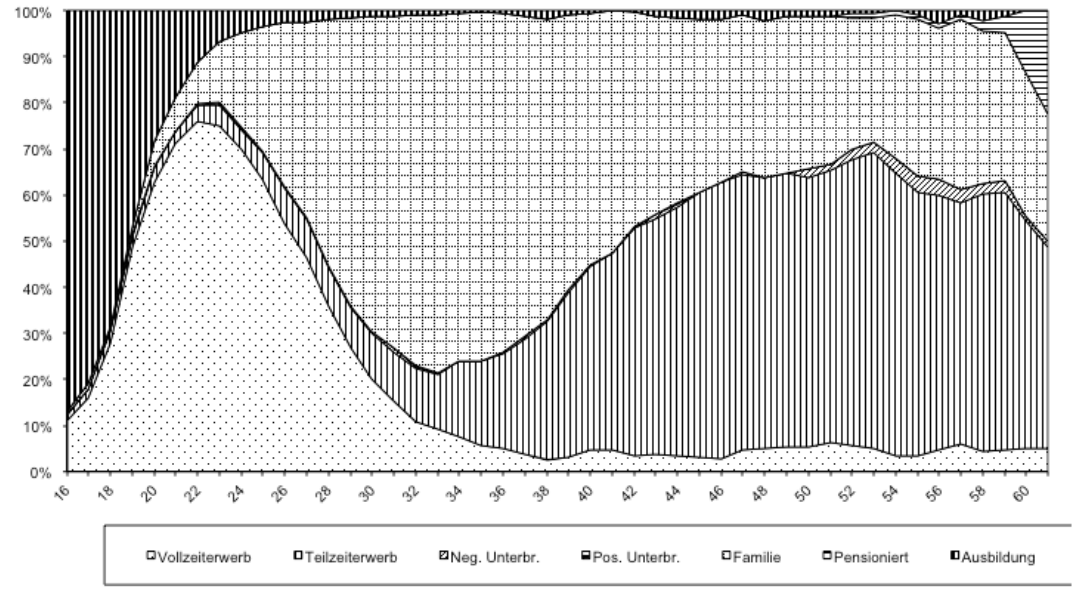

Es gibt also deutlich typisierte Verläufe, einen vorherrschenden für Männer und vier für Frauen, von denen einer mehr oder weniger dem männlichen gleicht, die anderen gehäuft nur bei 
Frauen vorkommen. Vergleicht man diese Befunde mit den drei oben erwähnten Thesen, so zeigt sich, dass keine von ihnen zutrifft.

1) Lebensläufe sind standardisiert - Beck hat nicht recht,

2) sie sind geschlechtsspezifisch - Kohli hat nicht recht,

3) es gibt zwar nur einen klar typisierten Verlauf bei Männern, aber vier bei Frauen - Levy hat auch nicht (völlig) recht.

Die gefundenen Verlaufstypen belegen eine stark vorherrschende Arbeitsmarktbeteiligung auch von Müttern, kombiniert mit variabler, aber zumindest ansatzweise in allen weiblichen Verlaufstypen vorkommender weiblicher Übernahme des Gros der Familienarbeit. ${ }^{9}$ Ohne dass die Typen selbst dies klar zum Ausdruck bringen könnten, erscheint es als höchst plausibel, dass die je nach Typ unterschiedlich weit gehende einseitige Rückkehr der Frau zur vorwiegenden Familienarbeit durch die Gegenwart von kleinen Kindern bedingt ist.

Ausgehend von den gefundenen Verlaufstypen stellt sich die Frage, welche Faktoren beeinflussen, in welchem dieser "Kanäle" weibliche Lebensläufe führen. Tabelle 1 (linker Teil) enthält die Analysen, mit den dies abgeklärt wurde. Da bei Männern nur zwei Verlaufskategorien gefunden wurden, genügt eine einfache Regressionsanalyse des ersten Verlaufstyps; die Analyse der zweiten Kategorie ergäbe einfach das umgekehrte Spiegelbild der Resultate der ersten. Gezeigt werden odds ratios, die angeben, um wie viel stärker oder schwächer eine Ausprägung im Vergleich zur Referenzkategorie ist. Lesebeispiel: bei besonders tiefen Haushaltseinkommen (1000-40'000 Fr.) ist die Wahrscheinlichkeit, dass der Mann einen Vollzeiterwerbsverlauf durchläuft, weniger als halb so gross wie in der Referenzkategorie, die hier die nächsthöhere Einkommensklasse darstellt.

Insgesamt fällt auf, dass nicht sehr viele Werte signifikant sind, was mit dem zahlenmässigen Vorherrschen und der damit verbundenen relativ geringen Varianz dieses Verlaufstyps unter Männern zusammenhängt. Beim Haushalteinkommen spielt nur die besonders tiefe Einkommenskategorie eine besondere Rolle, hier sind Verläufe mit Vollzeiterwerbstätigkeit deutlich seltener als in allen anderen Einkommensstufen. Bildung spielt dagegen eine nichtlineare Rolle, bei tiefer wie bei hoher Bildung sind Vollzeiterwerbsverläufe seltener als bei mittlerer Bildung. Das Alter hat dagegen keinen signifikanten Einfluss auf die männlichen Erwerbsverläu-

${ }^{9}$ Nebenbei gesagt bestätigen unsere vier Typen weitgehend die pragmatische Typologie, die Ende der 80er Jahre der Studie von Borkowsky und Streckeisen (1989, vgl. auch Borkowsky et al. 1985) zugrunde gelegt wurde: Doppelarbeiterinnen (Vollzeit) - Dreiphasenfrauen (Rückkehr) - Wechslerinnen (mehrfaches Hin- und Her, hier ist die Uebreinstimmung nur partiell) - Familienfrauen (Aufgabe der Erwerbstätigkeit). 
fe. Einen starken Einfluss hat dagegen das Vorhandensein von Kindern, besonders von mehreren, in diesen Fällen kommt der unter Männern ohnehin vorherrschende Vollzeiterwerbsverlauf noch verstärkt vor, er scheint hier gleichsam unumgänglich zu werden. Von den Zivilständen ist es nur das Ledigsein, das die Wahrscheinlichkeit eines Vollzeiterwerbsverlaufs deutlich senkt. Obwohl diese Koeffizienten die statistischen Einflüsse jeder einzelnen Variablen grundsätzlich ohne den Variationsanteil aufzeigt, der auf die anderen zurückzuführen ist, erscheint hier doch eine deutliche Interaktion zumindest zwischen Ledigsein und keine Kinder haben vorzuliegen, zu der möglicherweise auch noch das hohe Bildungsniveau gehört. Das würde bedeuten, dass spät heiratende und Vater werdende Männer, möglicherweise wegen verlängerter Ausbildungsdauer, erst mit entsprechender biographischer Verzögerung ins vorherrschende Verlaufsmuster einschwenken.

Tabelle 1 (rechter Teil) zeigt die analoge Analyse der weiblichen Verläufe, wobei das Vorliegen von vier Verlaufstypen hier eine multinomiale Analyse nötig macht. Wiederum werden odds ratios gezeigt, die allerdings in diesem Fall doppelt referenziert sind. Unter den vier Typen ist der Familienverlauf Referenzkategorie.

Tab. 1 : Logistische Regression (odds ratios) der vorherrschenden Verlaufstypen auf ausgewählte soziodemographische Variablen

\begin{tabular}{|c|c|c|c|c|}
\hline & Männer & \multicolumn{3}{|c|}{ Frauen } \\
\hline & $\begin{array}{c}\text { Referenzkategorien } \\
=1\end{array}$ & \multicolumn{3}{|c|}{$\mathrm{n}\left(\begin{array}{c}\text { Referenzkategorien unabhängige Variab } \\
\text { len }=1, \text { Referenzkategorie abhängige } \\
\text { Variable: Familienverlauf })\end{array}\right.$} \\
\hline & Vollzeit & Vollzeiterw & ilzeiterwerb & Rückkehr \\
\hline \multicolumn{5}{|l|}{ Haushalteinkommen } \\
\hline 1000-40000 Fr. & $0.417 * *$ & 0.80 & 0.99 & 0.85 \\
\hline $40 ’ 001-70 ' 000$ Fr. (Ref.) & 1 & 1 & 1 & 1 \\
\hline 70’001-100'000 Fr. & 1.167 & 1.28 & 1.02 & 1.18 \\
\hline $100^{\prime} 001-180^{\prime} 000 \mathrm{Fr}$. & 1.133 & $1.62 *$ & 1.12 & 1.01 \\
\hline \multicolumn{5}{|l|}{ Bildung $^{10}$} \\
\hline tief & $0.638 *$ & $0.59 * *$ & 1.06 & $0.59 * *$ \\
\hline mittel (Ref.) & 1 & 1 & 1 & 1 \\
\hline hoch & $0.569 * *$ & 1.02 & $2.97 * *$ & 0.88 \\
\hline \multicolumn{5}{|l|}{ Alter } \\
\hline $30-39$ & 1.017 & 1.48 & $1.80 *$ & $1.65^{*}$ \\
\hline $40-49$ & 1.314 & 0.95 & 0.90 & 0.92 \\
\hline 50-59 (Ref.) & 1 & 1 & 1 & 1 \\
\hline $60+$ & 1.359 & $0.52 * *$ & $0.44 * *$ & $0.49 * *$ \\
\hline \multicolumn{5}{|l|}{ Kinder } \\
\hline keine (Ref.) & 1 & 1 & 1 & 1 \\
\hline eines & 0.789 & 1.09 & 1.69 & 1.69 \\
\hline
\end{tabular}

${ }^{10}$ Als tief gilt ausschliesslich obligatorische Bildung, als mittel nachobligatorische Bildung unter Universitätsniveau, als hoch Universitätsoder Fachhochschulabschluss. 


\begin{tabular}{|l|c|ccc|} 
zwei & 1.250 & $\mathbf{0 . 3 2} * *$ & 0.86 & 1.03 \\
drei oder mehr & $\mathbf{1 . 6 6 1 * *}$ & $\mathbf{0 . 1 9 * *}$ & $\mathbf{0 . 4 9 * *}$ & 0.66 \\
Berufsstellung Vater & & & & \\
white collar & 0.941 & $\mathbf{0 . 6 5 *}$ & 1.02 & 1.16 \\
selbständig & 1.267 & 0.71 & 1.02 & 1.06 \\
qualifiziert (Ref.) & 1 & 1 & 1 & 1 \\
unqualifiziert & 0.948 & 1.10 & 1.15 & 1.23 \\
Bauer & 1.206 & 1.06 & 0.72 & 1.08 \\
Zivilstand & & & & \\
ledig & $\mathbf{0 . 5 2 1 *}$ & $\mathbf{4 5 . 5 1 * *}$ & $\mathbf{2 3 . 8 5 * *}$ & $\mathbf{1 0 . 2 4 *}$ \\
verheiratet (Ref.) & 1 & 1 & 1 & 1 \\
geschieden, getrennt & 1.173 & $\mathbf{1 3 . 5 0 * *}$ & $\mathbf{7 . 9 1 * *}$ & $\mathbf{6 . 8 0 * *}$ \\
verwitwet & 0.692 & 1.41 & 1.45 & 1.34 \\
\hline
\end{tabular}

Signifikante Werte fett gedruckt; $*=\mathrm{p}<.05, * *=\mathrm{p}<.01$

Tab. 1 weist den Vollzeiterwerbsverlauf als besonders selten aus bei Frauen mit zwei oder mehr Kindern, mit lediglich elementarer Ausbildung, mit mittlerer sozialer Herkunft, und in der höchsten Alterskategorie; dagegen kommt er verstärkt vor bei unverheirateten und in geringerem Ausmass auch bei verwitweten Frauen sowie bei höheren Haushalteinkommen. Dieser Verlaufstyp ist besonders stark im Geflecht der benützten unabhängigen Variablen verortet: sechs von 18 Koeffizienten sind hoch signifikant, zwei weitere signifikant. Mit anderen Worten ist sein Vorliegen besonders stark von der sozialen Stellung der Frauen bedingt, und in diesem Sinn erscheint er auch als der spezifischste. Er wird stark gefördert durch die Abwesenheit einer Familie (ledig, keine Kinder), ein hohes Bildungsniveau und junges Alter (allerdings nicht signifikant). Diese Situation scheint entweder aus einer besonders hohen Priorität zu resultieren, welche eine Frau ihrer Berufskarriere einräumt (einschliesslich Verzicht auf ein Familienleben oder zumindest seine Verschiebung) oder aus dem Ausschluss aus der Familienpartizipation durch Scheidung oder Trennung.

Der Teilzeiterwerbsverlauf wird durch hohe Bildung und junges Alter verstärkt, höheres Alter dagegen reduziert sein Vorkommen beträchtlich. Das Vorhandensein eines Kindes verstärkt ihn ebenfalls, während er von drei oder mehr Kindern stark reduziert wird. Soziale Herkunft und Haushalteinkommen spielen keine Rolle. Dagegen ist dieser Verlaufstyp besonders häufig bei unverheirateten Frauen, aber in einem gewissen Ausmass auch bei geschiedenen oder getrennt lebenden. Seine positionale Verankerung ist geringer als im Fall des Vollzeiterwerbsverlaufs (fünf hoch signifikante und ein signifikanter Koeffizient), bleibt aber dennoch ansehnlich.

Rückkehrverläufe kennzeichnen hauptsächlich Frauen mittleren Bildungsniveaus. Haushalteinkommen und soziale Herkunft sind nicht relevant, auch nicht die Zahl der Kinder, aber 
wenig unter der Signifikanzschwelle zeichnet sich eine Tendenz zur Abnahme mit der Kinderzahl ab; sie sind am häufigsten bei Müttern eines Kindes. Junge Frauen haben diesen Verlaufstyp besonders oft, ältere besonders selten. Wie die beiden vorhergehenden Typen ist auch dieser besonders häufig bei unverheirateten Frauen und auch bei Witwen. Er ist besonders wenig durch die unabhängigen Variablen beeinflusst (drei hoch signifikante Koeffizienten, zwei signifikante), was darauf hinweist, dass er besonders unspezifisch bzw. allgemein ist, wenn auch nicht der häufigste.

Die Funktionsweise der multinomialen Regression erlaubt es, die Bedingungsstruktur des Verlaufstyps Familie als Spiegelbild der drei anderen Typen zu identifizieren. Dieser Typ tritt gehäuft auf bei Frauen mit nicht mehr als elementarer Schulbildung, bescheidener sozialer Herkunft und tiefem Haushalteinkommen, mit eher fortgeschrittenem Alter und mit mehreren Kindern, vor allem auch bei verheirateten Frauen. Separate (nicht multinomiale) Regressionsanalysen bestätigen diese Lesart.

Damit erscheinen die drei erwerbsorientierten Verlaufstypen von Tab. 1 auch von ihrer Bedingungsstruktur her als das Gegenstück des Familienverlaufs, denn grosso modo sind ihre Assoziationen mit Bildung, Alter, Kinderzahl und Zivilstand gleichgerichtet. Es scheint also einen allgemeinen, unseren Einzelbefunden zugrundeliegenden Orientierungsmechanismus zu geben, der die Hauptwahl zwischen Familien- und Erwerbsorientierung reguliert, und sekundäre Faktoren, die zwischen den drei Varianten von biographischer Erwerbsorientierung unterscheiden. ${ }^{11}$ Die Hauptentscheidung, nämlich zwischen Erhaltung oder Aufgabe der Berufstätigkeit, hängt in erster Linie von der Kinderzahl und auch vom Alter ab, aber nicht vom Haushalteinkommen, wie man erwarten könnte, während Bildung und soziale Herkunft eher die genauere Verlaufsform der Arbeitsmarktteilnahme beeinflussen.

Vergleicht man zum Abschluss dieser Analyse die Resultate für Frauen und Männer, so stellt man deutliche Unterschiede fest. Geringes Haushalteinkommen verstärkt erratische Verläufe bei Männern, beeinflusst aber weibliche Verläufe kaum (mit Ausnahme des Vollzeiterwerbstyps, der eine schwach signifikante lineare Beziehung mit Einkommen aufweist, welche der Hypothese widerspricht, die Erwerbstätigkeiten von Frauen und besonders von Müttern werde vor allem von finanziellen Zwängen diktiert). Bildung zeigt eine nichtlineare Beziehung zum Vollzeiterwerbsverlauf bei Männern mit einem Maximum bei mittlerem Bildungsniveau, wäh-

\footnotetext{
${ }^{11}$ Gewisse AutorInnen sprechen diesbezüglich geradeheraus von individuellen Wahlentscheidungen, besonders Hakim (1998, 2000). Wir können diese Interpretation nicht teilen, weil unsere Resultate (wie auch jene vieler anderer Studien) auf die massgeblichen Einflüsse sozialstruktureller Faktoren hinweisen. Solche Einflüsse widersprechen einer rein individualistischen und aktorialen Interpretation der vorhandenen Optionen (Crompton \& Harris 1998a, 1998b; Blossfeld \& Drobnič 2001; Ernst et al. 2009).
} 
rend sie bei Frauen als jener Faktor erscheint, der neben der Kinderzahl am stärksten zwischen den Verlaufstypen unterscheidet: je höher das Bildungsniveau, desto stärker engagieren sich die Frauen in Teilzeiterwerbsverläufen, je tiefer, desto stärker durchlaufen sie Familienverläufe. Alter ist ebenfalls von einiger Bedeutung für weibliche Verläufe, nicht aber für männliche. Die Gegenwart und Zahl von Kindern drückt Männer verstärkt in Vollzeiterwerbsverläufe, Frauen dagegen noch deutlicher in Familien- und Teilzeitwerbsverläufe, vor allem wenn es zwei oder mehr Kinder sind. Auch der Zivilstand ist wesentlich wichtiger für weibliche als für männliche Verläufe, während soziale Herkunft für beide Geschlechter keine grosse Rolle spielt.

Insgesamt erscheint der Einfluss sozialer und vor allem sozialstruktureller Faktoren für weibliche Verläufe schwerwiegender als für männliche, und weibliche (Erwerbs-) Verläufe reagieren viel stärker und differenzierter auf die - über Zeit, d.h. über den Familienzyklus hinweg variablen Erfordernisse des Familienlebens als männliche.

\subsection{Statusgewichtung: geschlechtsspezifische Masterstatus}

Was ergibt sich aus diesem empirischen Exkurs zum Thema der Gewichtung unterschiedlicher Statusbereiche? Die Resultate entsprechen dem Konzept der geschlechtsspezifischen Masterstatus (Krüger und Levy 2000), welches die heutige, sozusagen postparsonianische Familien- und Geschlechterverfassung beschreibt und hier nur knapp erwähnt sei: bei Männern ist Berufsteilnahme und Erfüllung ihrer Erfordernisse dominant, Partizipationen an anderen Feldern werden durch diese begrenzt, auch an der Familie - bei Frauen ist invers die Familienteilnahme und die Erfüllung derer Erfordernisse dominant, Partizipationen an anderen Feldern werden durch diese begrenzt, auch an der Berufswelt. Diese anscheinende Symmetrie zwischen den Geschlechtern enthält soziologisch gesehen eine deutliche Ungleichheit, weil damit in den meisten Fällen die Frauen wirtschaftlich und in einem weiteren Sinn statusmässig (Prestige, Lebensstil, soziales Kapital...) von ihrem Mann abhängig werden, und weil sie einseitig den grösseren Teil der Familienarbeit übernehmen.

\section{3 Übergang zur Elternschaft}

Die berichteten Resultate weisen deutlich darauf hin, dass der Übergang zur Elternschaft einen Bruch in der Familienorganisation bedeutet, wenn auch in unterschiedlichem Ausmass. Auch vorher egalitär funktionierende Paare traditionalisieren augenscheinlich ihre Struktur, d.h. die Statusprofile der Partner, wozu vor allem der teilweise oder definitive Rückzug der 
Frau vom Arbeitsmarkt gehört, aber ebenso die kompromisslos vollzeitliche Erwerbsintegration des Mannes.

Ausgehend von dieser Feststellung stellt sich die Frage, wie dieser Umbruch in den Paaren zustande kommt: wird er antizipiert oder nicht, können die Paare ihre diesbezüglichen Absichten realisieren oder passen sie diese Absichten der äusseren Realität an?

Dazu einige erste Resultate aus der Studie "Devenir parent", die in der Romandie zugleich quantitativ und qualitativ durchgeführt wurde, und zwar als Panel mit drei Wellen (Le Goff und Levy 2011): 1. Befragungswelle ungefähr im 8. Schwangerschaftsmonat, 2. Welle 1 Monat nach der Geburt, und 3. Welle 10 Monate später. Die Analysen sind zwar im Moment der Abfassung dieses Textes noch im Gange, aber vier Feststellungen zeichnen sich schon deutlich ab (Abbildung 7):

1. Junge Paare, die Eltern werden, teilen weitgehend den Wert der Gleichheit von Frau und Mann (untere Graphiken, Welle 1: über die Hälfte der Partner beider Geschlechter geben egalitäre Absichten zur Aufteilung der Hausarbeit an). ${ }^{12}$

2. Während der Schwangerschaft entsprechen ihre Werte über die (egalitäre) Verteilung der verschiedenen Elemente der Familienarbeit weitgehend ihrer diesbezüglichen Praxis (obere Graphiken, Welle 1: deutlich über die Hälfte der Paare weisen höchstens zwei von vier Hausarbeiten geschlechtstypisch ungleich $\mathrm{zu}) .{ }^{13}$

\footnotetext{
${ }^{12}$ Direkte Frage, analog für Männer und Frauen: "Lorsque votre enfant sera né, comment envisagez-vous, après votre congé maternité (ou quand votre enfant aura environ 6 mois, pour les femmes qui n'ont pas de congé maternité), de vous répartir les tâches ménagères entre vous et votre conjoint/compagnon?", Antwortkategorien recodiert (je ferai presque tout / je ferai les trois quarts / je ferai la moitié / je ferai le quart / je ferai moins).

${ }^{13}$ Der Traditionalismus-Index zählt einfach, wieviele von vier Hausarbeiten im Paar geschlechtsstereotyp zugeteilt sind, z.B. bedeutet stark traditionell, dass drei der vier Arbeiten traditionell zugeordnet sind). Die Arbeitskategorien sind Putzen, Waschen, Kochen, Einkaufen. Korrelationen zwischen Absichten und Praktiken in der 1. Welle bestätigen diese Feststellung.
} 
Abb. 7 Arbeitsteilung im Paar: berichtete Praktiken (oben) und Absichten (unten) aus der Sicht der Frauen und der Männer

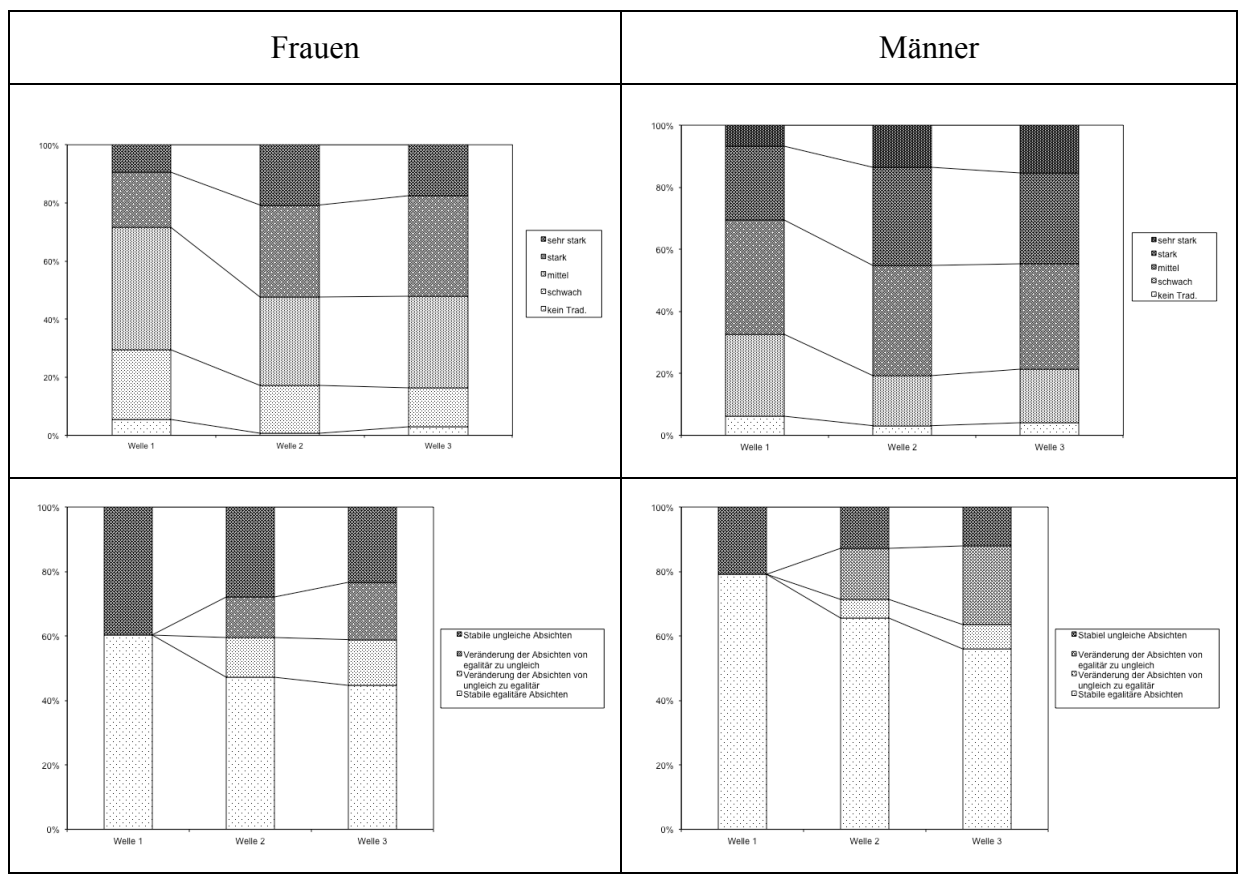

3. Ihr effektives Verhalten nach der Geburt bzw. nach dem Mutterschaftsurlaub entspricht nicht diesen Werten und Absichten, sondern in erster Linie der neuen Situation mit einem Säugling, und verschieben sich in Richtung der traditionellen Rollenverteilung (obere Graphiken, 3 Wellen: der Anteil traditionell geschlechtsstereotyper Aufgabenzuteilung nimmt über die Wellen deutlich zu, nach Angabe der Männer noch stärker als nach Angabe der Frauen).

4. Die so entstehende Kluft zwischen Werten und Verhalten wird allmählich (nur teilweise von der 1. zur 2., teilweise erst von der 2. zur 3. Befragungswelle) durch Anpassung der Normen und Werte reduziert, die ihrerseits nach traditional verschoben werden (bei den Frauen erfolgt die Hauptanpassung bereits vom ersten zum zweiten Befragungszeitpunkt, bei den Männern erfolgt ein ebenso deutlicher zusätzlicher Anpassungsschub zwischen dem zweiten und dem dritten Zeitpunkt). ${ }^{14}$

\footnotetext{
${ }^{14}$ Eine solche Diskrepanz tritt dagegen in Bezug auf etwa die Absichten, die Grosseltern fürs Kinderhüten zu mobilisieren, nicht auf.
} 
Allgemein entsteht somit ein Bild, nach dem junge Paare, wenn sie Eltern werden, in Handlungszwänge geraten, die nicht ihren Absichten entsprechen und deren Ausmass sie nicht genügend antizipieren. Dies macht plausibel, dass die Retraditionalisierung ihrer Familie mehr durch exogene Zwänge als durch eine rein interne Dynamik zustande kommt. Diese Interpretation legt die weitere Hypothese nahe, dass die Institutionen, welche das Alltagsleben der Familien rahmen (Arbeitswelt, Stundenpläne von Krippen und Schulen, Konsumrhythmen, Öffnungszeiten und Benützungsbedingungen von Ämtern und öffentlichen Diensten wie Gesundheit u.ä.) so funktionieren, dass sie weitgehend die Verfügbarkeit einer erwachsenen Person pro Familie voraussetzen, welche sich auf die verschiedenen Aspekte von Familienarbeit spezialisiert. Dass dies im allgemeinen die Frau und Mutter ist, hängt sicher nicht nur, aber auch von der Frauendiskrimination auf dem Arbeitsmarkt ab, die es für Paare zumindest finanziell gesehen rational macht, eher ganz oder teilweise auf den Lohn der Frau als auf jenen des Mannes zu verzichten.

Dieses Gesamtbild verweist auf den institutionellen Rahmen der Gesellschaft und spezifischer auf das schweizerische Sozialstaatsmodell - ein weitläufiges Thema, das hier nur höchst knapp angeschnitten werden kann. Geht man von den drei Haupttypen von Sozialstaat aus, die aus der Forschungstradition von Esping-Andersen $(1990,1999)$ hervorgegangen sind, so gehört die Schweiz recht eindeutig zur liberalen Variante, jedenfalls was ihre Familien- und Genderpolitik betrifft (Levy 2007). Diese ist durch starke Zurückhaltung bei staatlichen Interventionen geprägt, d.h. eine ausgesprochen restriktive Interpretation des Subsidiaritätsprinzips. Das bestätigt von der Ergebnisseite her der internationale Vergleich der Altersverläufe der weiblichen Berufstätigkeit (Maruani 1993, 2003), aus dem ebenfalls drei Verlaufsmodelle resultieren, die recht gut den drei Esping-Andersen-Typen entsprechen: ein skandinavisches Modell, das die Vereinbarkeit von Familie und Beruf für Frauen ausdrückt, d.h. es besteht kein fundamentaler Unterschied zwischen männlichen und weiblichen Erwerbsverläufen; ein familistisches, südeuropäisches Modell, nach dem bereits Heirat und Beruf für Frauen unvereinbar sind; und ein liberal-individualistisches Modell mit einem Doppelbuckel, das Länder wie Deutschland und die Schweiz kennzeichnet, bei dem Mutterschaft und Beruf einander tendenziell ausschliessen. Abbild 8 schematisiert diese drei Modelle. 
Abb. 8 Modelle weiblicher Erwerbsverläufe, europäische Länder (nach Maruani 1993, 2003)
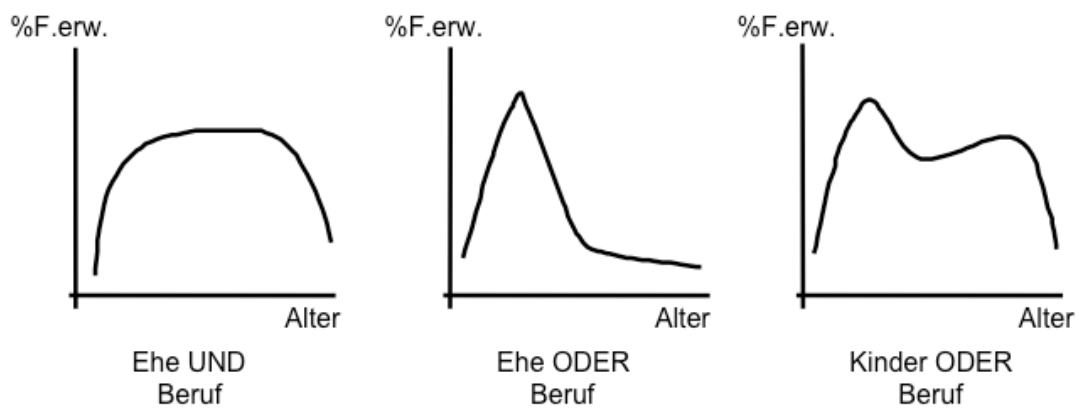

Will man gegenüber diesem weitgehend institutionellen Doing gender eine emanzipatorische Perspektive entwickeln, so finden sich alle nötigen Ingredienzien im 7. deutschen Familienbericht (Bertram et al. 2006), der auf der gleichzeitigen Notwendigkeit von drei Arten von Massnahmen besteht:

- eine der gesellschaftlichen Komplexität angemessene Zeitpolitik,

- finanzielle Mittel, die genderneutral zugeteilt werden,

- institutionelle Strukturen familienergänzender Kinderbetreuung, die ohne Diskrimination über die Kaufkraft (d.h. hohe Preise) zugänglich sind.

Die institutionelle Ähnlichkeit von Deutschland und der Schweiz erlaubt es, trotz aller Unterschiede davon auszugehen, dass dieselben Schlussfolgerung auch für die Schweiz gültig sind.

\section{Zusammenfassung und Ausblick}

Ausgehend von einer vorwiegend, aber nicht ausschliesslich strukturtheoretischen Konzeption des Lebenslaufs als variabel institutionalisierter Bewegung durch den sozialen Raum, die analytisch als Sequenz von Statusprofilen gefasst werden kann, wurden empirische Verlaufsdaten aus dem Schweizerischen Haushaltpanel mittels Optimal Matching miteinander verglichen und regressions-analysiert. Dabei hat sich eine überraschend deutliche, wenn auch nuancierte Geschlechtstypik männlicher und weiblicher Lebensläufe herausgestellt, mit einem klar vorherrschenden Verlaufstyp bei Männern und vier Verlaufstypen bei Frauen, von denen einer 
einigermassen dem männlichen Standardtyp entspricht. Die nur bei Frauen vorkommenden Verlaufstypen zeichnen sich durch unterschiedliche und verschieden weit gehende Muster der Beeinflussung des Erwerbsverlaufs durch die Familiengeschichte aus, also durch den Übergang zur Elternschaft und das Aufwachsen der Kinder mit deren eigenen Statuspassagen (z.B: Kindergarten, Einschulung). Eine analoge Beeinflussung des männlichen Erwerbsverlaufs existiert kaum, der einzige systematische Einfluss ist schwächer als bei Frauen und geht in Richtung einer Konsolidierung des Standardmodells. Der Übergang zur Elternschaft erweist sich in der Analyse sozusagen als "Hauptschalter" dafür, dass die Frau, nicht aber der Mann, ihren Erwerbsverlauf der erhöhten Familienarbeit anpasst, sie einseitig übernimmt und damit de facto den Mann entlastet. Nach welchem Muster sie dies tut, hängt vor allem von familiären und individuellen Statuspositionen ab (Bildung, Beruf, Einkommen).

Diese empirisch belegte Geschlechtstypik der Erwerbsverläufe zeichnet ein scharfes Bild dessen, was besonders in der deutschen Soziologie als doppelte Vergesellschaftung der Frau, nicht aber des Mannes, bezeichnet wurde (Becker-Schmidt 1987), und zwar so, wie sie sich im konkreten institutionellen Kontext der Schweiz darstellt. Hochentwickelte Gesellschaften unterscheiden sich diesbezüglich, d.h. in Bezug auf ihr institutionalisiertes Genderregime, beträchtlich, wobei die drei Wohlfahrtsstaatstypen von Esping-Andersen $(1990,1999)$ sich als relevant herausstellen. Während Länder des sozialdemokratischen Typs (vorwiegend skandinavische Länder) deutlich und sichtlich erfolgreich ihre Familien- und Sozialpolitik auf Geschlechtergleichheit ausrichten, stellen Länder des konservativen Typs (z.B. Frankreich) die Unterstützung der Familie in den Vordergrund, wogegen liberale (angelsächsische Länder, in Sachen Genderregime aber weitgehend auch die Schweiz) die Staatsinterventionen stark beschränken - in der Schweiz u.a. unter dem Stichwort der Subsidiarität - und das Geschehen dem Markt, mit anderen Worten der individuellen Kaufkraft und den entsprechenden sozialen Ungleichheiten überlassen (Levy 2007).

Die vertiefte Analyse des Überganges zur Elternschaft zeigt, dass die Geburt des ersten Kindes für Familien im schweizerischen Kontext (ähnlich wie in Deutschland, aber anders als in Ländern der beiden anderen Wohlfahrtsstaatstypen) mit einer markanten Retraditionalisierung der Familienstruktur verbunden ist, die im Kontrast zu den egalitären Tendenzen junger Paare vor der Elternschaft steht. Dabei stellt sich auch heraus, dass viele Paare offensichtlich die Schwierigkeiten und Zwänge nicht im vollen Ausmass vorhersehen, mit denen sie sich auseinandersetzen müssen, sobald Kinder da sind. Der Vergleich der zeitlichen Veränderung der effektiven Arbeitsteilung im Paar mit den diesbezüglichen Absichten der PartnerInnen weist 
deutlich darauf hin, dass die erwähnten Zwänge stärker wirken als die Absichten, oder anders gesagt: die beobachtete Retraditionalisierung resultiert nicht in erster Linie aus entsprechenden Wünschen der Partner, sondern aus praktischen Handlungszwängen, die sie erst voll entdecken, wenn sich die Situation verändert hat - und viele reagieren, oft mit Verzögerung, indem sie ihre Wertvorstellung an ihre veränderte Praxis anpassen und sie ebenfalls zu verstärktem Traditionalismus verschieben. Nicht zu vergessen ist, dass zur Retraditionalisierung der Familienstruktur im Verlauf ihrer individuellen Geschichte auch die zunehmende wirtschaftliche Abhängigkeit der Frau von ihrem Mann gehört; die geschlechtsspezifische Differenzierung der Lebensläufe beinhaltet also auch die Dimension der Geschlechterungleichheit in Bezug auf die individuelle Stellung in der Gesellschaft.

Aus grösserer Distanz betrachtet erweist sich an diesen Befunden die Nützlichkeit der eingangs vorgestellten theoretischen Perspektive, die Statusübergängen oder Transitionen eine besonders grosse Bedeutung zuschreibt, ebenso die analytische Tragfähigkeit des Konzepts komplementärer geschlechtsspezifischer Masterstatus (Krüger \& Levy 2000). Auch die grössere Erklärungskraft strukturtheoretischer gegenüber akteur- und agency-theoretischer Ansätze zeichnet sich in den vorgestellten Resultaten zumindest ab - Menschen tun nicht einfach, was sie möchten, sondern meistens das, was ihnen die Möglichkeiten und Zwänge nahelegen, die sie in ihrem sozialen Umfeld vorfinden.

Vom Gesagten ausgehend können viele weitergehende Fragen entwickelt werden, sei es im direkten Zusammenhang mit dem Konzept des Statusprofils, sei es im konkreten Zusammenhang der sozialen Organisation geschlechtsdifferenter Lebensläufe. Vom Statusprofil ausgehend stellen sich etwa Fragen danach, wie sich dessen verschiedene Elemente zueinander verhalten, ob sie z.B. Rollenwidersprüche nach sich ziehen, oder welche Verhaltenstendenzen durch Statusinkonsistenzen stimuliert werden. Ein anderer Aspekt, der in der Forschung bisher kaum beachtet wurde, betrifft die mögliche Ausweitung oder Einschränkung des Statusprofils im Lebensverlauf durch Hinzufügen neuer Felder sozialer Teilnahme oder umgekehrt Aufgabe einer vorhandenen Teilnahme - was bedeuten diese Veränderungen für das Individuum, etwa im Sinn einer Intensivierung oder Abschwächung ihrer sozialen Integration?

Konkreter im Zusammenhang mit der Institutionalisierung oder allgemeiner der sozialen Organisation von Lebensläufen ist es interessant, dieses Institutionalisierungsgeschehen näher zu analysieren und theoretisch zu konzeptualisieren. Dabei wäre etwa zwischen direkter und indirekter Institutionalisierung zu unterscheiden: nur wenige Institutionen, die Auswirkungen 
auf Lebensverläufe haben, haben dies auch zum Ziel (das wäre der Fall der Schule für die SchülerInnen: sie bereitet offiziell auf das Erwachsenenleben vor und stattet die Menschen mit symbolischen Ressourcen dafür aus). Die übrigen verfolgen andere Ziele, wobei aber ihrem Funktionieren Normalitätsvorstellungen über ihre MitarbeiterInnen oder KundInnen zugrunde liegen, wie etwa jene, dass jede Familie über ein Mitglied verfügt, dass die nötigen Einkäufe während der üblichen Öffnungszeiten tätigen und sich dafür an die entsprechenden Orte begeben kann. Je unumgänglicher die Teilnahme an solchen Institutionen ist (etwa an der Arbeitswelt oder am Konsummarkt, oder an der Schule mit ihren "löchrigen" Stundenplänen für die Kinder), desto grösser ist der Druck auf die Teilnehmenden, ihre Lebensverhältnisse diesen impliziten Normalitätsmodellen anzugleichen, weil das Abweichen von ihnen etwa die partnerschaftliche Reduktion der Erwerbszeit im Paar - mit Kosten verbunden sind, die sich schnell als untragbar erweisen können. Weiter wäre es wichtig, mittels Mehrebenenanalyse auch empirisch die vorgestellten Interpretationen über den Einfluss des institutionellen Umfelds auf die konkreten Arrangements in den Paaren zu überprüfen, wie es etwa Elcheroth et al. (2011) angefangen haben.

\section{Bibliographie}

Aisenbrey, Silke. 2000. Optimal Matching Analyse. Anwendungen in den Sozialwissenschaften. Opladen: Leske + Budrich.

Bauman, Zygmut. 2000. Liquid Modernity. Cambridge: Polity Press.

Beck, Ulrich. 1986. Risikogesellschaft. Auf dem Weg in eine andere Moderne. Frankfurt/ Main: Suhrkamp.

Beck, Ulrich und Elisabeth Beck-Gernsheim (Hrsg.). 1994. Riskante Freiheiten. Individualisierung in modernen Gesellschaften. Frankfurt/Main: Suhrkamp.

Becker-Schmidt, Regina (1987): Die doppelte Vergesellschaftung - die doppelte Unterdrückung: Besonderheiten der Frauenforschung in den Sozialwissenschaften. In: Lilo Unterkircher und Ina Wagner (Hrsg.): Die andere Hälfte der Gesellschaft. Österreichischer Soziologentag 1985. Soziologische Befunde zu geschlechtsspezifischen Formen der Lebensbewältigung. Wien: Verlag des Österreichischen Gewerkschaftsbundes, 10-25.

Bernath, Walter, Martin Wirthensohn \& Erwin Löhrer. 1989. Jugendliche auf ihrem Weg ins Berufsleben. Bern: Haupt.

Bertram, Hans, Helga Krüger und C. Katharina Spiess. 2006. Familie zwischen Flexibilität und Verlässlichkeit. Perspektiven für eine lebenslaufbezogene Familienpolitik. Siebter Familienbericht. Berlin: Bundesministerium für Familie, Senioren, Frauen und Jugend.

Blancpain, Robert \& Erich Häuselmann. 1974. Zur Unrast der Jugend. Frauenfeld: Huber.

Blossfeld, Hans-Peter \& Sonja Drobnič. 2001. Theoretical Perspectives on Couples' Careers. in: Blossfeld, Hans-Peter \& Sonja Drobnič (eds.), Careers of Couples in Contemporary Society. From Male Breadwinner to Dual-Earner Families. Oxford: Oxford University Press, 16-50. 
Borkowsky, Anna, Elisabeth Kästli, Katharina Ley und Ursula Streckeisen. 1985. Zwei Welten - ein Leben. Berichte und Anregungen für Frauen zwischen Familie und Beruf. Zürich: Unionsverlag.

Borkowsky, Anna und Ursula Streckeisen. 1989. Arbeitsbiographien von Frauen. Eine soziologische Untersuchung objektiver und subjektiver Aspekte. Grüsch: Rüegger.

Born, Claudia \& Helga Krüger (Hrsg.). 2001. Individualisierung und Verflechtung. Geschlecht und Generation im deutschen Lebenslaufregime. München: Juventa.

Bourdieu, Pierre. 1980. Questions de sociologie. Paris: Minuit. (dt. Soziologische Fragen. Berlin: Suhrkamp 1993.)

Crompton, Rosemary \& Fiona Harris 1998. A Reply to Hakim. British Journal of Sociology 49(1): 144-149.

Diewald, Martin, Anne Goedicke \& Karl Ulrich Mayer (eds.). 2006. After the Fall of the Wall: Life Courses in the Transformation of East Germany. Palo Alto: Stanford University Press.

Elcheroth, Guy, Felix Bühlmann \& Manuel Tettamanti. 2011. Valeurs égalitaires et pratiques sexuées: une approche biographique et comparative. in: Dominique Joye, Christine Pirinoli, Dario Spini \& Eric Widmer (dir.), Parcours de vie et insertions sociales. Zürich: Seismo 81-104.

Elder, Glen H. 1974. Children of the Great Depression: Social Change in Life Experience. Chicago: University of Chicago Press.

Ernst, Michèle \& René Levy. 2002. Lebenslauf und Regulation in Paarbeziehungen : Bestimmungsgründe der Ungleichheit familialer Arbeitsteilung. Zeitschrift für Familienforschung 14(2), 103-131.

Esping-Andersen, Gøsta. 1990. The Three Worlds of Welfare Capitalism. Cambridge: Polity Press.

Esping-Andersen, Gøsta. 1999. Social Foundations of Postindustrial Economics. New York: Oxford University Press.

Giddens, Antony. 1991. Modernity and Self-Identity. Self and Society in the Late Modern Age. Cambridge: Polity Press.

Hakim, Catherine. 2000. Work-Lifestile Choices in the 21st Century: Preference Theory. New York : Oxford University Press.

Hakim, Catherine. 1998. Developing a sociology for the twenty-first century: preference theory. British Journal of Sociology 49(1): 137-143.

Heintz, Peter. 1968. Einführung in die soziologische Theorie. Stuttgart: Enke.

Heinz, Walter, Johannes Huinink und Ansgar Weymann (Hrsg.). 2009. The Life Course Reader. Individuals and Societies Across Time. Frankfurt/Main: Campus.

Held, Thomas \& René Levy. 1974. Die Stellung der Frau in Familie und Gesellschaft. Eine soziologische Analyse am Beispiel der Schweiz. Frauenfeld: Huber.

Hradil, Stefan. 1990. Postmoderne Sozialstruktur? Zur empirischen Relevanz einer "modernen" Theorie sozialen Wandels. In Lebenslagen, Lebensläufe, Lebensstile. Hrsg. Peter A. Berger und Hradil, 125-150, Stefan. Soziale Welt, Sonderband 7.

Kluge, Susanne \& Udo Kelle (Hrsg.). 2001. Methodeninnovation in der Lebenslaufforschung. Integration qualitativer und quantitativer Verfahren in der Lebenslauf- und Biographieforschung. München: Juventa.

Kohli, Martin. 1985. Die Institutionalisierung des Lebenslaufs. Kölner Zeitschrift für Soziologie und Sozialpsychologie, 37(1): 1-29. 
Kohli, Martin. 1986. Gesellschaftszeit und Lebenszeit. Der Lebenslauf im Strukturwandel der Moderne. Soziale Welt (Sonderband "Die Moderne - Kontinuitäten und Zäsuren"): 183208.

Kohli, Martin. 2003. Der institutionalisierte Lebenslauf: ein Blick zurück und nach vorn. In Entstaatlichung und soziale Sicherheit. Verhandlungen des 31. Kongresses der DGS. Hrsg. Jutta Allmendinger, 525-545. Opladen: Leske+Budrich.

Krüger, Helga. 2001. Geschlecht, Territorien, Institutionen. Beitrag zu einer Soziologie der Lebenslauf-Relationalität. In Individualisierung und Verflechtung. Geschlecht und Generation im deutschen Lebenslaufregime. Hrsg. Claudia Born \& Helga Krüger, 257299. München: Juventa.

Krüger, Helga und René Levy. 2000. Masterstatus, Familie und Geschlecht. Vergessene Verknüpfungslogiken zwischen Institutionen des Lebenslaufs. Berliner Journal für Soziologie 10(3): 379-401.

Le Goff, Jean-Marie und René Levy. 2011. Le Goff J.-M. \& Levy R. (im Druck). Liens entre intentions et pratiques au moment de la transition à la parentalité. Quelques résultats de l'enquête Devenir parent. Actes du colloque de l'Association des conseillers d'orientation-psychologues. Les parcours sociaux. Entre nouvelles contraintes et affirmation $d u$ sujet. Rennes: Presses universitaires de Rennes.

Leisering, Lutz, Rainer Müller \& Karl F. Schumann (Hrsg.). 2001. Institutionen und Lebensläufe im Wandel. Institutionelle Regulierungen von Lebensläufen. München: Juventa.

Levy, René. 1977. Der Lebenslauf als Statusbiographie. Die weibliche Normalbiographie in makrosoziologischer Perspektive. Stuttgart: Enke.

Levy, René. 2007. Particulière, singulière ou ordinaire ? La régulation suisse des parcours de vie sexués. In Sonderfall Schweiz. Hrsg. Thomas Eberle und Kurt Imhof, 226-247. Zurich: Seismo.

Levy, René, Jacques-Antoine Gauthier und Eric Widmer. 2006. Entre contraintes institutionnelle et domestique: les parcours de vie masculins et féminins en Suisse. Cahiers canadiens de sociologie 31(4): 461-489.

Levy, René, Paolo Ghisletta, Jean-Marie Le Goff, Dario Spini und Eric Widmer (Hrsg.). 2005. Towards an Interdisciplinary Perspective on the Life Course. (Advances in Life Course Research 10). Amsterdam, Boston etc.: Elsevier.

Lewin, Kurt. 1951. Field Theory in Social Science. New York: Harper Bros. (deutsch: Feldtheorie in den Sozialwissenschaften. Ausgewählte theoretische Schriften. Huber, Bern/Stuttgart 1963)

Linton, Ralph. 1936. The Study of Man. New York: Appleton-Century.

Lyotard, Jean-François. 1979. La condition post-moderne. Rapport sur le savoir. Paris: Editions de Minuit.

Maruani, Margaret. 1993. L'Emploi dans l'Europe des Douze. Bruxelles: Commission des Communautés Européennes.

Maruani, Margaret. 2003. Travail et emploi des femmes. Paris: La Découverte.

Merton, Robert K. 1957. Social Theory and Social Structure. Glencoe, IL: Free Press.

Sackmann, Reinhold \& Matthias Wingens (Hrsg.). 2001. Strukturen des Lebenslaufs. Uebergang - Sequenz - Verlauf. München, Juventa.

Thoits, Peggy A. 1986. Multiple Identities: Examining Gender and Marital Status Differences in Distress. American Sociological Review 51(2): 259-272. 
Thomas, William I. \& Florian Znaniecki. 1918. The Polish Peasant in Europe and America. Chicago: Chicago University Press.

West, Candace \& Don H. Zimmerman. 1987. Doing gender. Gender and society 1(2): 125151.

Widmer, Eric, René Levy, Alexandre Pollien, Raphaël Hammer und Jacques-Antoine Gauthier. 2003a. Entre standardisation, individualisation et sexuation: une analyse des trajectoires personnelles en Suisse. Revue suisse de sociologie 29(1): 35-67.

Widmer, Eric, Jean Kellerhals, René Levy, Michèle Ernst und Raphael Hammer. 2003b. Couples contemporains - Cohesion, régulation et conflits, une enquête sociologique. Zurich: Seismo. 